\title{
Research of Oil Well Working Parameters Monitoring System Based on Internet of Things
}

\author{
Lei Wenli ${ }^{1,2, a}$, Wang Fubao ${ }^{1}$, Ren Xincheng ${ }^{2}$ \\ ${ }^{1}$ School of Electronics and Information, Northwestern Polytechnical University, Xi'an, 710072, \\ China; \\ ${ }^{2}$ College of Physic and Electronic Information, Yan'an University, Yan'an 716000, China \\ alei.wenli@163.com
}

Keywords: Internet of things; oil well; working parameters; monitor;

\begin{abstract}
In view of the current oil collection points are most remote and scattered, the existing mobile communication network are often not covered, it is difficult to realize automatic monitoring for working condition of oil well, this paper presents a oil well working parameters monitoring system based on the Internet of things, which can realize the automatic wireless acquisition and monitoring of the wellhead pressure, temperature and displacement parameters etc. The system consists of a collection of nodes, routing nodes, gateway nodes, local networks and cloud computing platform. Collection nodes are developed on core processor ARM7 and embedded uClinux operating system platform, the working parameters data of the oil well is sent to the local area network and cloud computing platform through wireless network. Monitoring personnel can view the real-time working parameters of oil well in the local network which provides an important support for the protection of oil well safety and accident rescue.
\end{abstract}

\section{Introduction}

Oil resources as an important national resource, occupy an important position in the energy structure. At present, oil monitoring technology is still mainly with common manual monitoring and wired network monitoring in domestic ${ }^{[1]}$. As the oil well collection point most remote and work sites scattered, it has causing a lot of problem, higher installation cost, big difficulty for wiring and maintenance management, long monitoring period, the large amount of data, high labor intensity, low efficiency, targeted poor, low accuracy and so on ${ }^{[2]}$. This paper presents a kind of oil well working parameters monitoring system based on the Internet of things, which can realize the automatic wireless collection and monitoring of the wellhead pressure, oil temperature, displacement parameters displacement and other working parameters. The system consists of a collection of nodes, routing nodes, gateway nodes, local networks and cloud computing platform. Monitoring personnel can view the real-time working parameters of oil well in the local network which provides an important support for the protection of oil well safety and accident rescue.

\section{Internet of Things Technology}

The concept of things put forward in 1999, refers to form a sensor network by various information sensing device, such as a radio frequency identification device, sensor node, GPS, laserscanner, camera, embedded communication module, and transmission of the various information of the physical world which has acquired via a communication network to centralized information processing and application platform. oil well wireless network monitoring system based on the Internet of things is proposed in this paper which is to form the Internet of things at the blind area of the existing mobile communication network, collect the work parameters of oil well, and will send the collected data to the local area network, the local network is responsible for access cloud computing network by wired or wireless means, it not only greatly saves cost, but also solves the interruption problem of monitoring system for the collection point is located at the mobile communication blind area. 


\section{Design of Oil Well Monitoring System}

Overall Structure of oil well monitoring system as shown in Figure 1, the whole system consists of oil well production parameters collection network and background data supporting platform two part. Oil well production parameters collection network is mainly composed of site collection nodes, routing nodes and gateway nodes. Data supporting platform is responsible for backstage data handling, displaying, and feeding back the results to the production site after analysis and processing.

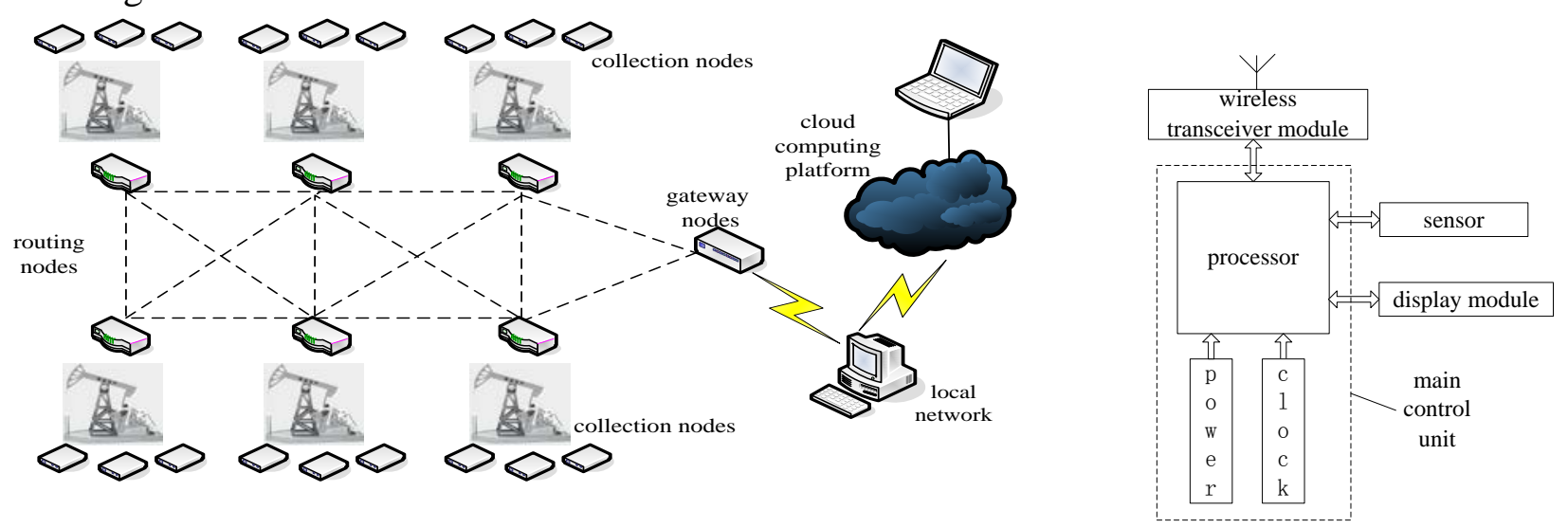

Fig.1. System composition

Fig.2. Hardware block diagram of Collection node

\section{Hardware Design of Collection Node}

The hardware of oil well working parameters collection node includes main control unit, sensor, wireless transceiver module and display module ${ }^{[3]}$, the hardware block diagram is shown in figure 2.

The main control unit is used to ensure the normal acquisition of oil wellhead working parameters data through the sensing element, maintaining the normal operation of a system, controlling data transmission of wireless transceiver module, packaging information data and so on, which hardware circuit is composed of processor, power and clock circuit. Because the processor is the core component of the collection node, it need has powerful ability of processing data, by comparing, it select the S3C44B0 microprocessor which suitable for using as the main control chip of oil well working parameters collection node. The power supply is composed of two $5 \mathrm{~V}$ dry battery.

Due to work in the wild for a long time, the wireless transceiver module need has the advantages of low power consumption and high reliability. Monolithic RF transceiver chip nRF2401 which working in 2.4 2.5GHz ISM band is selected here ${ }^{[4]}$.

According to which the working parameters of the oil well to be monitored, the sensor module are chosen current sensor, pressure sensor, load sensor, displacement sensor and temperature sensor five sensor, respectively for acquisition of the motor current, the wellhead pressure, linkage rod load, temperature of collection point and other data.

\section{Software Design}

The software of oil well working parameters collection node includes system software and applications program two parts. Operating system is the basic platform to run applications, where embedded uClinux software platform is used. uClinux software platform is consisted of the bootloader, kernel image file and file system ${ }^{[5]}$.

System Transplantation. Bootloader is a section of the initialization code program running in front of uClinux operating system ${ }^{[6]}$, is responsible for initializing part of hardware equipment, address mapping, loading the uClinux kernel image file to memory, guiding embedded uClinux to start. Bootloader can direct view, modify physical address space and burn-in the uClinux kernel and file system in command line mode in the later stage of program maintenance and development. The 
oil well working parameters collection node chooses the Blob as the boot program, combining the system software and hardware, and leading the uClinux operating system. Blob source code is completely open, followed by the GPL. It can start the uClinux kernel, also can be used for simple debugging, which is a powerful bootloader.

Module design is adopted by uClinux kernel, that is, a lot of function block can be independently added or removed, which can select the desired function module of the system and remove unwanted features at compile time. Through the reconfiguration to the kernel, it can make the kernel of the systems significantly reduced, thus reducing resource usage. This paper selects the Linux2.4 kernel, using the graphical kernel configuration interface and cross compiler tool chain arm-elf-gcc compile, adding the system support for ARM7 processors.

The uClinux kernel image file of elf format can be generated in the program folder under /arm/arch/ after compiling. And then the kernel file will be burned into the flash through the serial port or network.

In the uClinux operating system platform, it uses romfs as the file system of the oil well working parameters collection nodes, including the application, configuration files and library files. Compared to other file system, romfs file system can save more system space.

Application Development. The application program of oil well working parameters collection node is written by interface functions provided by uClinux operating system platform, mainly to complete collecting, transmitting and displaying work parameters of oil well. The software of data acquisition tasks include: motor current data acquisition, wellhead pressure acquisition, a linkage rod load acquisition, the wellhead temperature acquisition. After power on, the terminal system initialization is first completed, including the initialization of serial port, setting baud rate, data bits, stop-bit number, and software or hardware flow control; then, it starts the power saving mode, detects serial instruction; when it detects acquisition command, then starts configuration of wireless transceiver module, establishes wireless communication, at the same time starts the receiving sub-thread (receiving data from the sensing element), sending sub-thread, displaying sub-thread. When the system fails to receive the data acquisition command, then, it is pending acquisition task, starting power saving task and entering the power saving mode. Software process is as shown in figure 3(1). After starting acquisition thread, A/D transforming is carried by system at first, and then calculating the working parameters of the oil well, until receiving thread is finished, as shown in figure 3(2). When sending thread starts, the system first to determine whether there have data need to sent, if yes, then sending data until the process is finished, as shown in figure 4(1). When displaying thread starts, firstly the system will get the data which will be displayed, and then send it to display by LCD, as shown in figure 4(2).

\section{Conclusion}

This paper completed the design of a monitoring system for the oil well working parameters based on the Internet of things, the system hardware and software of the oil well working parameters collection node is described in detail. ARM7 embedded processor is used for processing of collection data, controlling wireless transceiver module and display module in this design. The powerful computing ability and low power consumption characteristics of ARM7 processor, can satisfy the working environment of long-term field. Using ZigBee wireless transceiver module, the system achieves the data communication between collection nodes, routing nodes and gateway. It has the widespread practical value in the protection of oil production safety and management, and provides technical reference for the construction of digital oilfield and intelligent oilfield. 


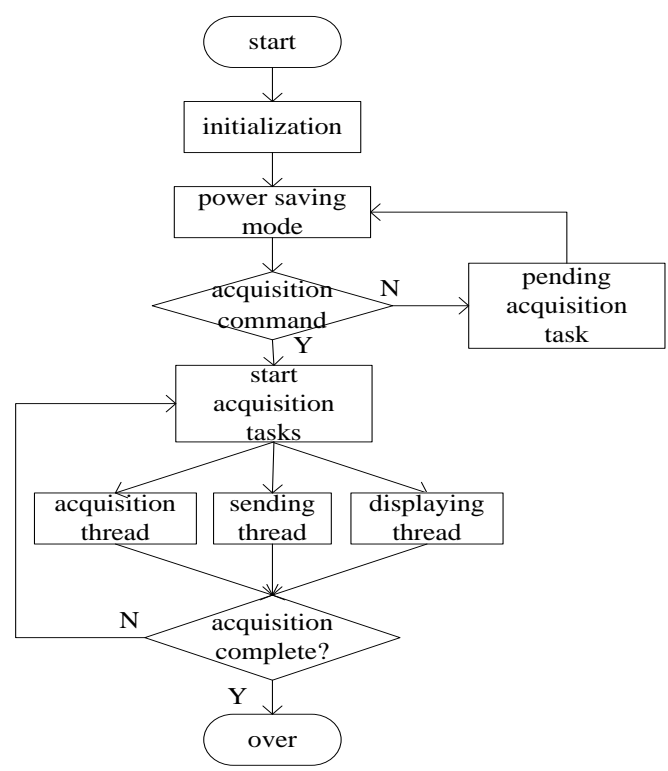

(1)

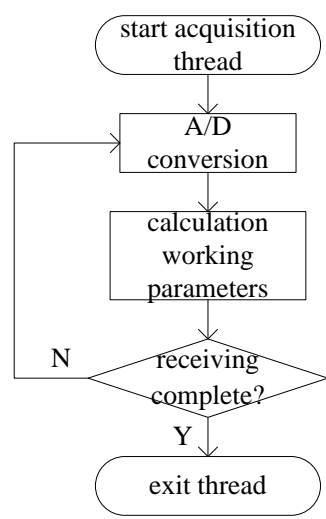

(2)

Fig.3. (1) The software flow chart of oil well working parameters collection nodes

(2) The software flow chart of collecting thread

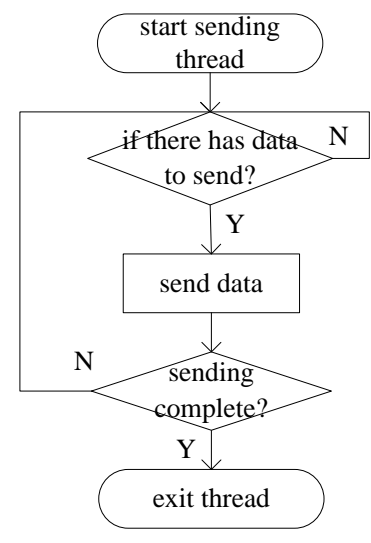

(1)

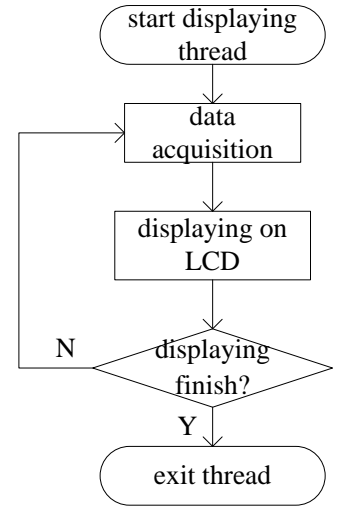

(2)

Fig.4. (1) The software flow chart of sending thread

(2) The software flow chart of displaying thread

\section{Acknowledgements}

The authors acknowledge with thanks the Scientific Research Program Funded by Shaanxi Provincial Education Department (Program No. 14JK1829), Yan'an Municipal Science and Technology Research and Development Program(Program No. 2014KG-04), the Science and Technology Research and Development Program Project in Shaanxi Province (Industrial Research) (Grant No. 2014K05-61), and the National Nature Science Foundation of China (Grant No. 61379026).

\section{References}

[1] Huo Qiuzhen. Research and design of remote monitoring system of oil well based on wireless sensor network[D]. Nanjing: Nanjing University of Science and Technology, 2011.04

[2] Chen Yongjun, Liao Dezhang. Design of oil pumping machine remote monitoring system based on dsPIC digital signal controller[J]. Journal of Yangtze University (NATURAL SCIENCE EDITION), 2010.12, 122-134.

[3] Li na. Multi channel data acquisition system of oil well[J]. Oil and gas field surface engineering, 2014.01, 63-64. 
[4] Wang Ji, Xu Gongbao, Shen Yuli. Water Heavy Metal Monitoring System Based on Wireless Sensor[J]. Computer Measurement and Control. 2009.04. 643-645

[5] Mu Naigang. Zigbee Technical Overview[J]. Telecommunications Technology. 2006.03. 84-86

[6] Sun Yi. Basis For The Development of Embedded Systems[M]. Xi'an University of Electronic Science and Technology Press. 2008.08 